\title{
Método das Diferenças Finitas Energéticas para simulação do comportamento sob flexão do concreto reforçado com fibras de aço
}

\author{
Júlia Barbosa Neves ${ }^{(1)}$, José Mário Feitosa Lima ${ }^{(2)}$, Paulo Roberto Lopes Lima ${ }^{(3)}$
}

\begin{abstract}
Resumo
Apesar do uso cada vez mais frequente de fibras de aço em elementos estruturais de concreto armado, ainda é incipiente o desenvolvimento de modelos teóricos ou computacionais que possam ser utilizados para o dimensionamento e análise desses elementos. A adição de fibras de aço altera o comportamento tensão-deformação do concreto, tanto na compressão quanto na tração, sendo que, neste último caso, o material mantém uma tensão residual pós-fissuração, mesmo a grandes deformações. Portanto, torna-se necessário a utilização de diagramas tensão-deformação diferenciados que incorporem a contribuição das fibras de aço para prever mais acuradamente o comportamento estrutural de elementos constituídos desse material. No presente trabalho é apresentada uma formulação variacional com base no Método das Diferenças Finitas Energéticas (MDFE) para a previsão do comportamento à flexão de vigas de concreto armado, combinando a teoria clássica de laminados (TCL) com um modelo de dano aplicado ao concreto. A validação da proposta é feita com base em resultados carga-deslocamento, experimentais e teóricos, encontrados na literatura para vigas de concreto reforçadas com três teores de reforço em fibras de aço.
\end{abstract}

Palavras-chave: Fibra de aço. Viga de concreto. Modelo de dano de Mazars. Laminados. Método das diferenças finitas energéticas.

1 Engenheira Civil, Mestrado em Engenharia Civil e Ambiental/DTEC, Universidade Estadual de Feira de Santana, Av. Transnordestina, SN, Novo Horizonte, 44030900, Feira de Santana, Bahia, Brasil. julianeves@gmail.com

2 Professor Adjunto, Mestrado em Engenharia Civil e Ambiental/DTEC, Universidade Estadual de Feira de Santana, Av. Transnordestina, SN, Novo Horizonte, 44030900, Feira de Santana, Bahia, Brasil. lima.jmf@gmail.com

3 Professor Titular, Mestrado em Engenharia Civil e Ambiental/DTEC, Universidade Estadual de Feira de Santana, Av. Transnordestina, SN, Novo Horizonte, 44030900, Feira de Santana, Bahia, Brasil. Lima.prl@pq.cnpq.br

http://dx.doi.org/10.5335/rsee.v10i2.2667 


\section{Introdução}

A adição de fibras curtas de aço ao concreto tem permitido o desenvolvimento de um material com maior capacidade de absorção de energia. As fibras interligam as microfissuras e reduzem a velocidade de propagação das mesmas, o que faz com que a ruptura aconteça a deformações muito maiores que as esperadas para o concreto simples. Além disso, enquanto no concreto sem fibras a região fissurada é considerada isenta de tensões, no concreto com fibras há uma transferência de esforços entre as duas faces da fissura o que resulta em manutenção de carregamento de tração mesmo após o surgimento da primeira fissura (RILEM, 2002). Como resultado, a adição de fibras de aço contribui para um aumento da deformação de ruptura de vigas de concreto simples (Soranakom, Mobasher, 2007) e aumento da resistência à flexão de vigas de concreto armado (Massicote, 2003). Com relação ao cisalhamento, verifica-se que a adição de $2 \%$ de fibras de aço aumentou a resistência ao cisalhamento e modificou a forma de ruptura da viga de concreto armado (Furlan Jr, Hanai, 1997).

As vantagens do uso de fibras de aço em concreto têm difundido sua utilização como reforço secundário em estruturas de concreto (Bentur, Mindess, 2007) e mesmo como reparo de estruturas (Ruano et al, 2012). No entanto, devido à mudança no comportamento tensão-deformação sob tração e compressão, a utilização de fibras de aço não é prevista na norma brasileira de dimensionamento de estruturas de concreto armado, visto que as leis constitutivas existentes na NBR 6118 (ABNT, 2008) são aplicadas apenas para concreto simples.

Neste trabalho é avaliada a possibilidade de aplicação, para concreto reforçado com fibras de aço, da lei constitutiva proposta por Mazars e Lamaitre (1984), baseada na Mecânica do Dano e proposta inicialmente para concreto simples sob tração ou compressão. Para isso, um programa computacional baseado no Método das Diferenças Finitas Enérgéticas (MDFE) foi desenvolvido. Baseado no tradicional Método das Diferenças Finitas, que recentemente vem sendo utilizado para modelagem estruturas de concreto armado (Jones et al, 2010; Habib et al., 2010), o MDFE é um método rápido e preciso, que são características desejáveis no dimensionamento de estruturas.

\section{Relações constitutivas}

\subsection{Concreto simples}

No modelo proposto por Mazars e Lamaitre (1984) o comportamento do concreto sob tensão uniaxial é caracterizado por duas fases associadas ao dano do material (microfissuração interna). Na fase pré-fissuração $\left(\varepsilon \leq \varepsilon_{d 0}\right)$ o concreto apresenta um comportamento elástico linear sob tensão e o parâmetro de dano $D$ é nulo. Na fase pós-fissuração, quando a deformação $\varepsilon$ ultrapassa o limite de deformação elástica ( $\mathcal{E}>\varepsilon_{d 0}$ ) o módulo elástico inicial $E_{0}$ é progressivamente danificado, assumindo o valor $E$, de acordo com: 


$$
E_{C}=(1-D) E_{0}
$$

O parâmetro escalar de dano $D$ varia entre 0 e 1 e é calculado por:

$$
D=\left\{\begin{array}{c}
1-\frac{\varepsilon_{d 0}\left(1-A_{T}\right)}{\varepsilon_{e q}}-\frac{A_{T}}{\exp \left[B_{T}\left(\varepsilon_{e q}-\varepsilon_{d 0}\right)\right]}, \text { se } \varepsilon>\varepsilon_{d 0} \\
1-\frac{\varepsilon_{d 0}\left(1-A_{C}\right)}{\varepsilon_{e q}}-\frac{A_{C}}{\exp \left[B_{C}\left(\varepsilon_{e q}-\varepsilon_{d 0}\right)\right]}, \text { se } \varepsilon<-\frac{\varepsilon_{d 0}}{v \sqrt{2}}
\end{array}\right.
$$

onde $A_{T}, B_{T}, A_{C}, B_{C}$ são parâmetros que dependem do material, associados ao diagramas tensão-deformação, na tração e compressão, e $\mathcal{E}_{e q}$ é a deformação equivalente, dada por:

$$
\varepsilon_{e q}= \begin{cases}\varepsilon, & \varepsilon \geq 0 \\ -v \varepsilon \sqrt{2}, & \varepsilon<0\end{cases}
$$

onde $V$ é o coeficiente de Poisson do concreto.

\subsection{Concreto com fibras de aço}

A introdução de fibras curtas de aço no concreto implica em alteração no comportamento tensão-deformação, que é dependente do tamanho e volume de fibras. $\mathrm{Na}$ compressão a principal mudança está associada ao comportamento tensão-deformação após a carga de pico: é observado uma queda de resistência menos acentuada à medida que fibras de aço são inseridas no concreto (NATARAJA et al, 1999).

A principal modificação acontece, no entanto, no comportamento sob tração visto que o concreto simples é considerado frágil e após a introdução da fibra uma resistência residual é observada. As leis constitutivas são expressas em termos de tensão versus deformação ou em termos de tensão versus abertura de fissura. Pode-se utilizar leis constitutivas não-lineares (RILEM, 2002) ou, de forma mais simplificada, leis constitutivas linearizadas como as mostradas na Figura 1. 




a)

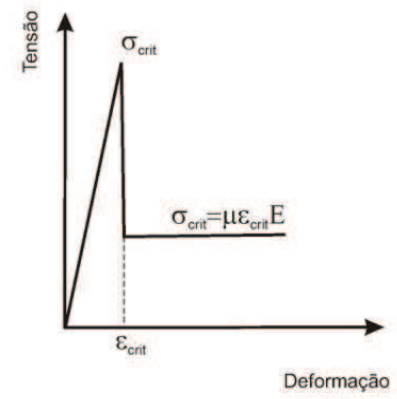

b)

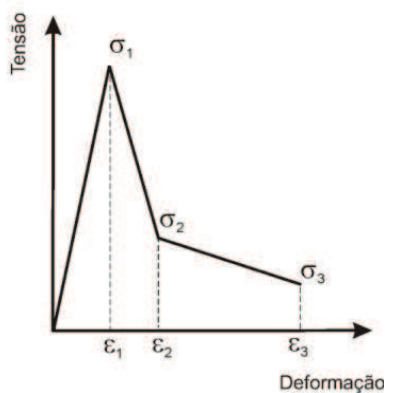

c)

Figura 1 - Curvas esquemáticas linearizadas para representação do comportamento pós-fissuração do concreto com fibras: a) RILEM (2002); Naaman e Reinhardt (2006); b) Soranakom e Mobasher (2007); c) Barros (1995); Grossi (2006)

\section{Formulação variacional para o problema}

\subsection{Hipóteses básicas e campo de deslocamentos}

As vigas em estudo são prismáticas, de seção retangular, e idealizadas como formadas por lâminas (ou camadas) de espessura uniforme. Aplicam-se as seguintes hipóteses:

a) durante a flexão as seções transversais permanecem planas e inextensíveis (hipótese de Euler-Bernoulli);

b) as deformações e rotações são assumidas pequenas perante a unidade (linearidade geométrica);

c) há aderência perfeita entre as camadas na zona de contato;

d) cada camada é constituída de apenas um tipo de material (camadas homogêneas, não compósitas);

e) as cargas e os vínculos são aplicados diretamente no eixo ${ }^{x}$ da viga.

Com base nas hipóteses (a), (b) e (c), tem-se o seguinte campo de deslocamentos (Figura 2):

$$
\begin{gathered}
u(x)=u_{0}(x)-z \frac{\partial w_{0}(x)}{\partial x} \\
v(x)=0 \\
w(x)=w_{0}(x)
\end{gathered}
$$


sendo $u(x), v(x), w(x)$ as componentes do deslocamento de um ponto genérico da viga segundo as direções $x, y$ e $z$, respectivamente, e $u_{0}(x)$ e $w_{0}(x)$ os deslocamentos segundo os eixos $x$ e $z$, nessa ordem, do ponto associado ao primeiro e localizado no eixo da viga.

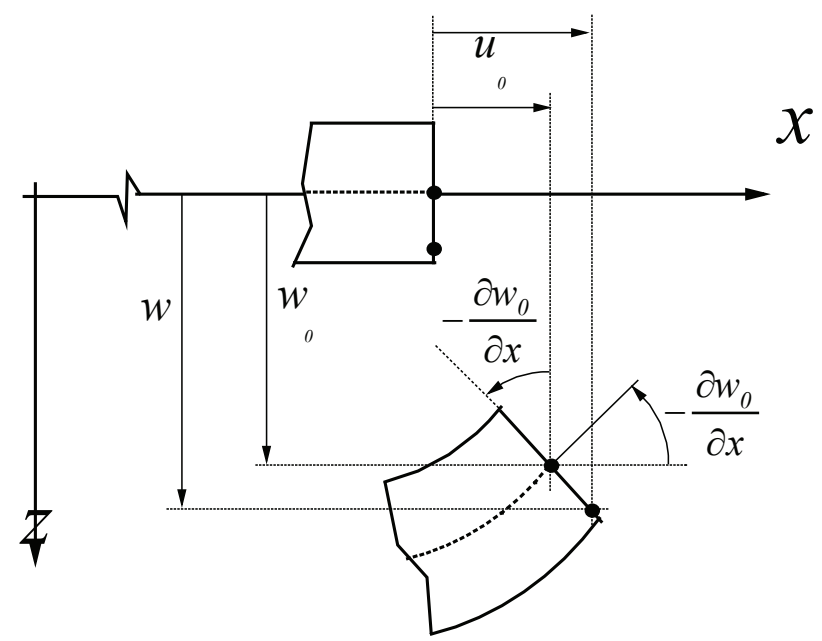

Figura 2 - Componentes de deslocamento e geometria de deformação

\subsection{Relações Tensão-Deformação}

As relações deformação-deslocamento para o problema, lineares de acordo com a hipótese (b), resultam nulas após a substituição do campo de deslocamentos (4), à exceção da componente $\mathcal{E}_{x}$, como ocorre na teoria clássica de vigas:

$$
\varepsilon_{x}=\frac{\partial u}{\partial x}=\frac{\partial u_{0}}{\partial x}-z \frac{\partial^{2} w_{0}}{\partial x^{2}}
$$

A Teoria Clássica de Laminados estabelece que as lâminas estão sob estado plano de tensões. Nesse contexto, com base na Lei de Hooke Generalizada, é válida a seguinte relação entre tensões e deformações em cada camada $k$ do laminado:

$$
\left\{\begin{array}{c}
\sigma_{x} \\
\sigma_{y} \\
\tau_{x y}
\end{array}\right\}=\left[\begin{array}{ccc}
Q_{11}^{(k)} & Q_{12}^{(k)} & 0 \\
Q_{12}^{(k)} & Q_{22}^{(k)} & 0 \\
0 & 0 & Q_{66}^{(k)}
\end{array}\right]\left\{\begin{array}{c}
\varepsilon_{x} \\
\varepsilon_{y} \\
\gamma_{x y}
\end{array}\right\}
$$


onde as grandezas $Q_{11}^{(k)}, Q_{12}^{(k)}, Q_{22}^{(k)}$ e $Q_{66}^{(k)}$ são constantes mecânicas relacionadas às propriedades de engenharia do material da camada $k$, conforme definido em Jones (1999) e Reddy (2004). Observando que a única componente de deformação não nula do modelo é $\mathcal{E}_{x}$, tem-se:

$$
\left\{\begin{array}{l}
\sigma_{x} \\
\sigma_{y} \\
\tau_{x y}
\end{array}\right\}=\left[\begin{array}{ccc}
Q_{11}^{(k)} & 0 & 0 \\
0 & Q_{22}^{(k)} & 0 \\
0 & 0 & Q_{66}^{(k)}
\end{array}\right]\left\{\begin{array}{c}
\varepsilon_{x} \\
0 \\
0
\end{array}\right\}=\left\{\begin{array}{c}
Q_{11}^{(k)} \varepsilon_{x} \\
0 \\
0
\end{array}\right\}
$$

acarretando, como também ocorre na Teoria de Vigas de Euler-Bernoulli, em uma única componente de tensão $\sigma_{x}$, dada por:

$$
\sigma_{x}=Q_{1}^{(k)} \varepsilon_{x}=\left(1-D^{(k)}\right) E^{(k)}
$$

onde $E^{(k)}$ representa o módulo de elasticidade longitudinal do material que constitui a camada $k$ e $D^{(k)}$ é a respectiva função de dano, lembrando que a hipótese (d) estabelece um único material por camada.

\subsection{Esforços Solicitantes}

$\mathrm{Na}$ formulação são requeridas as seguintes integrais de tensões na seção transversal genérica, de área $A$ :

$$
\begin{gathered}
N=\int_{A} \sigma_{x} d A=b \int_{-h / 2}^{h / 2} \sigma_{x} d z \\
M=\int_{A} \sigma_{x} z d A=b \int_{-h / 2}^{h / 2} \sigma_{x} z d z
\end{gathered}
$$

sendo $N$ o esforço normal, $M$ o momento fletor, $b$ e $h$ a largura e altura da seção.

Segundo a Teoria de Laminados a avaliação desses esforços é feita somando-se a contribuição oriunda de cada lâmina, processo no qual se considera os diferentes materiais (DANIEL; ISHAI, 2006). Portanto, substituindo nas equações (9) a relação constitutiva (8) da camada genérica $k$, e a deformação longitudinal $\varepsilon_{x}$ dada em (5), tem-se que: 


$$
\begin{aligned}
& N=b \frac{\partial u_{0}}{\partial x} \sum_{k=1}^{N C} Q_{11}^{(k)}\left(z_{k+1}-z_{k}\right)-\frac{b}{2} \frac{\partial^{2} w_{0}}{\partial x^{2}} \sum_{k=1}^{N C} Q_{11}^{(k)}\left(z_{k+1}^{2}-z_{k}^{2}\right) \\
& M=\frac{b}{2} \frac{\partial u_{0}}{\partial x} \sum_{k=1}^{N C} Q_{11}^{(k)}\left(z_{k+1}^{2}-z_{k}^{2}\right)-\frac{b}{3} \frac{\partial^{2} w_{0}}{\partial x^{2}} \sum_{k=1}^{N C} Q_{11}^{(k)}\left(z_{k+1}^{3}-z_{k}^{3}\right)
\end{aligned}
$$

com $N C$ simbolizando o número de lâminas que formam a seção, sendo cada lâmina $k$ delimitada pelas coordenadas $z_{k}$ e $z_{k+1}$, conforme Figura 3.
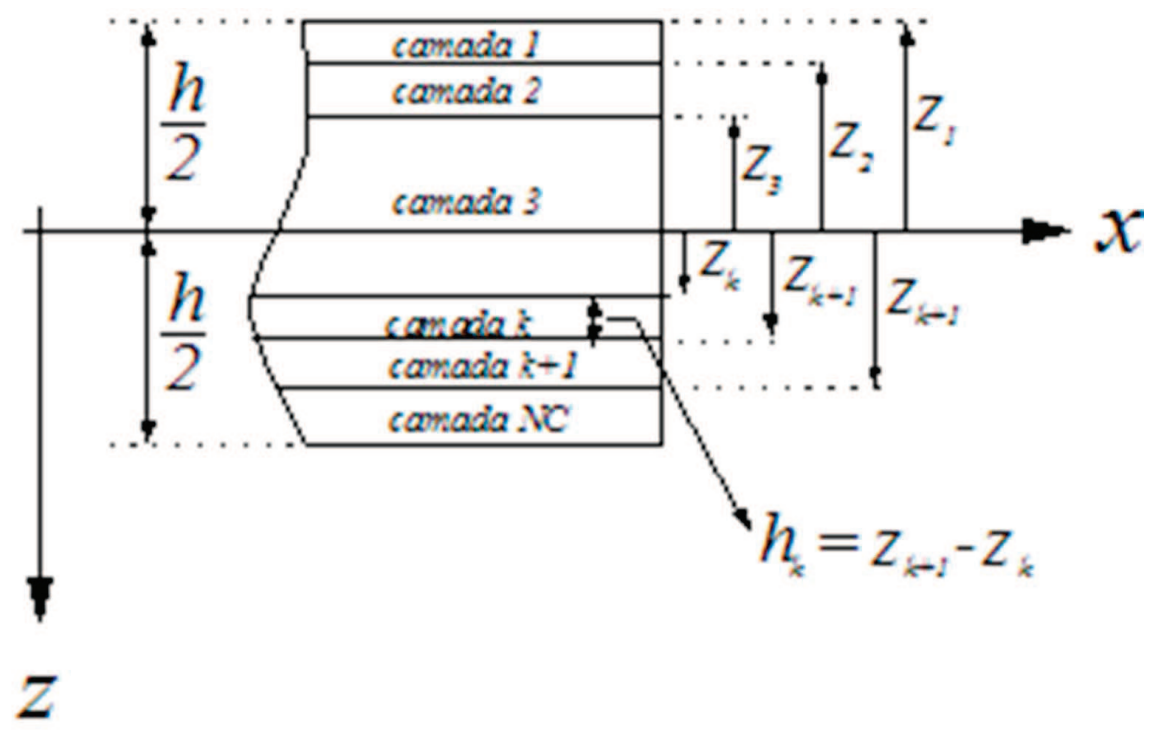

Figura 3 - Laminação da viga

O desenvolvimento dos somatórios em (10) conduz a expressões mais compactas para os esforços, quais sejam:

$$
\begin{aligned}
& N=A_{11} \frac{\partial u_{0}}{\partial x}-B_{11} \frac{\partial^{2} w_{0}}{\partial x^{2}} \\
& M=B_{11} \frac{\partial u_{0}}{\partial x}-D_{11} \frac{\partial^{2} w_{0}}{\partial x^{2}}
\end{aligned}
$$


onde

$$
\begin{aligned}
& A_{11}=b \sum_{k=1}^{N C} Q_{11}^{(k)}\left(z_{k+1}-z_{k}\right) \\
& B_{11}=\frac{b}{2} \sum_{k=1}^{N C} Q_{11}^{(k)}\left(z_{k+1}^{2}-z_{k}^{2}\right) \\
& D_{11}=\frac{b}{3} \sum_{k=1}^{N C} Q_{11}^{(k)}\left(z_{k+1}^{3}-z_{k}^{3}\right)
\end{aligned}
$$

cabendo associar às grandezas $A_{11}, B_{11} e D_{11}$, respectivamente, ao termo [11] das matrizes de rigidez extensional $[A]$ rigidez flexional $[B]$ e de acoplamento $[D]$, da Teoria de Laminados (Jones, 1999). É importante observar ainda que essas grandezas dependem diretamente de $Q_{11}^{(k)}$ que, de acordo com a equação (8), incorpora o dano ocorrido nas camadas, seja de concreto ou de aço (elásto-plástico).

\subsection{Princípio dos Trabalhos Virtuais}

O trabalho virtual realizado pelas forças internas $\delta W_{i n t}$ é dado por:

$$
\delta W_{\text {int }}=\int_{V} \sigma_{x} \delta \varepsilon_{x} d V
$$

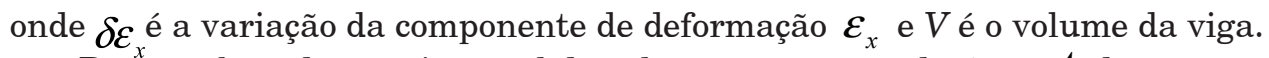

Desmembrando essa integral de volume em termos da área $A$ da seção e do comprimento $L$ da viga, e em seguida levando-se em conta as equações (5), de $\varepsilon_{x}$, e (11), dos esforços $N$ e $M$, tem-se para o trabalho virtual interno:

$$
\delta W_{\text {int }}=\int_{0}^{L}\left[\left(A_{11} \frac{\partial u_{0}}{\partial x}-B_{11} \frac{\partial^{2} w_{0}}{\partial x^{2}}\right) \delta\left(\frac{\partial u_{0}}{\partial x}\right)-\left(B_{11} \frac{\partial u_{0}}{\partial x}-D_{11} \frac{\partial^{2} w_{0}}{\partial x^{2}}\right) \delta\left(\frac{\partial^{2} w_{0}}{\partial x^{2}}\right)\right] d x
$$

O trabalho virtual realizado pelas forças externas, com base na hipótese (e) e na Figura 4, é dado por:

$$
\delta W_{e x t}=\int_{0}^{L}\left[p(x) \delta u_{0}+q(x) \delta w_{0}\right] d x+\left[\bar{F}_{x} \delta u_{0}+\bar{F}_{z} \delta w_{0}-\bar{M} \delta\left(\frac{\partial w_{0}}{\partial x}\right)\right]_{0}^{L}
$$


com $p(x)$ e $q(x)$ representando as cargas distribuídas de domínio, segundo as direções $x$ e $z$, respectivamente, e nas extremidades $x=0$ e $x=L$ da viga, estão aplicadas as forças segundo as direções $x$ e $z, \bar{F}_{x}$ e $\bar{F}_{z}$ respectivamente, e o momento $\bar{M}$ (as cargas foram desenhadas com o sentido positivo na Figura 4).

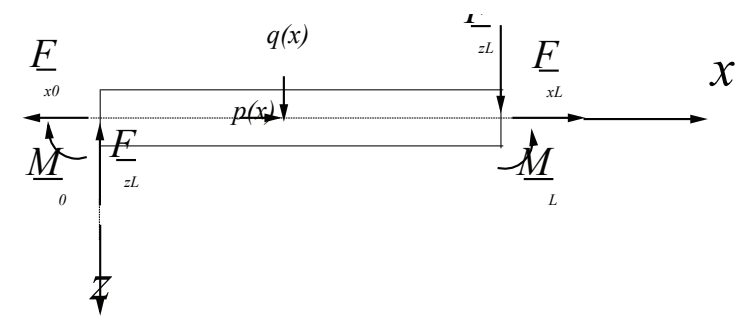

Figura 4 - Carregamento considerado na viga

Aplicando-se então o Princípio dos Trabalhos Virtuais (PTV), ou seja:

$$
\delta W_{i n t}=\delta W_{e x t}
$$

institui-se as equações diferenciais do problema:

$$
\begin{array}{lll}
A_{11} \frac{\partial^{2} u_{0}}{\partial x^{2}}-B_{11} \frac{\partial^{3} w_{0}}{\partial x^{3}}=-p(x) & \text { ou } & \frac{\partial N}{\partial x}=-p(x) \\
B_{11} \frac{\partial^{3} u_{0}}{\partial x^{3}}-D_{11} \frac{\partial^{4} w_{0}}{\partial x^{4}}=q(x) & \text { ou } & \frac{\partial^{2} M}{\partial x^{2}}=q(x)
\end{array}
$$

e as possíveis condições de contorno, em $x=0$ e $x=L$, adequadas ao modelo:

$$
\begin{gathered}
u_{0}=\bar{u}_{0} \quad \text { e } \quad \delta u_{0}=\delta \bar{u}_{0} \text { ou } N=\bar{F}_{x} \\
w_{0}=\bar{w}_{0} \quad \text { e } \quad \delta w_{0}=\delta \bar{w}_{0} \text { ou } Q=\bar{F}_{z} \\
\frac{\partial w_{0}}{\partial x}=\frac{\partial \bar{w}_{0}}{\partial x} \quad \text { e } \quad \delta\left(\frac{\partial w_{0}}{\partial x}\right)=\delta\left(\frac{\partial \bar{w}_{0}}{\partial x}\right) \text { ou } M=\bar{M}
\end{gathered}
$$


sendo $Q$ o esforço cortante na seção, cuja expressão é obtida a partir do equilíbrio de um elemento diferencial da viga, ou seja $Q=\frac{\partial M}{\partial x}=B_{11} \frac{\partial^{2} u_{0}}{\partial x^{2}}-D_{11} \frac{\partial^{3} w_{0}}{\partial x^{3}}$.

\section{Tratamento numérico do problema}

O tratamento em termos do método das diferenças finitas energéticas consiste em introduzir nas expressões das parcelas $\delta W_{i n t}$ e $\delta W_{\text {ext }}$, que compõem o princípio dos trabalhos virtuais (PTV), as representações em diferenças finitas utilizadas para as derivadas dos deslocamentos supondo, para fins de avaliação desses trabalhos virtuais, a barra subdividida em trechos de integração, ao longo dos quais todas as grandezas envolvidas são supostas constantes. Uma vez computados $\delta W_{i n t}$ e $\delta W_{\text {ext }}$, mediante o somatório das contribuições dos diversos trechos de integração, e considerando-se as condições de vinculação da barra, surge, pela aplicação do PTV, um sistema de equações algébricas de equilíbrio que, uma vez resolvido, permite obter a solução em termos dos deslocamentos nodais $u_{0}$ e $w_{0}$ incógnitos.

\subsection{Discretização}

A discretização adotada acha-se esquematizada na Figura 5. A barra é subdividida em $\mathrm{N}$ partes iguais, de comprimento $\lambda=L / \mathrm{N}$, totalizando $\mathrm{N}+1$ nós reais. Acrescentam-se ainda dois nós virtuais à barra, por conta da avaliação das derivadas primeira e segunda dos deslocamentos nas extremidades da barra. Portanto há $N$ nós na barra acarretando $N N-2$ trechos de integração. Na Figura 5 foram também representados os deslocamentos $u_{0}$ e $w_{0}$ numerados de acordo com um sistema de numeração global.

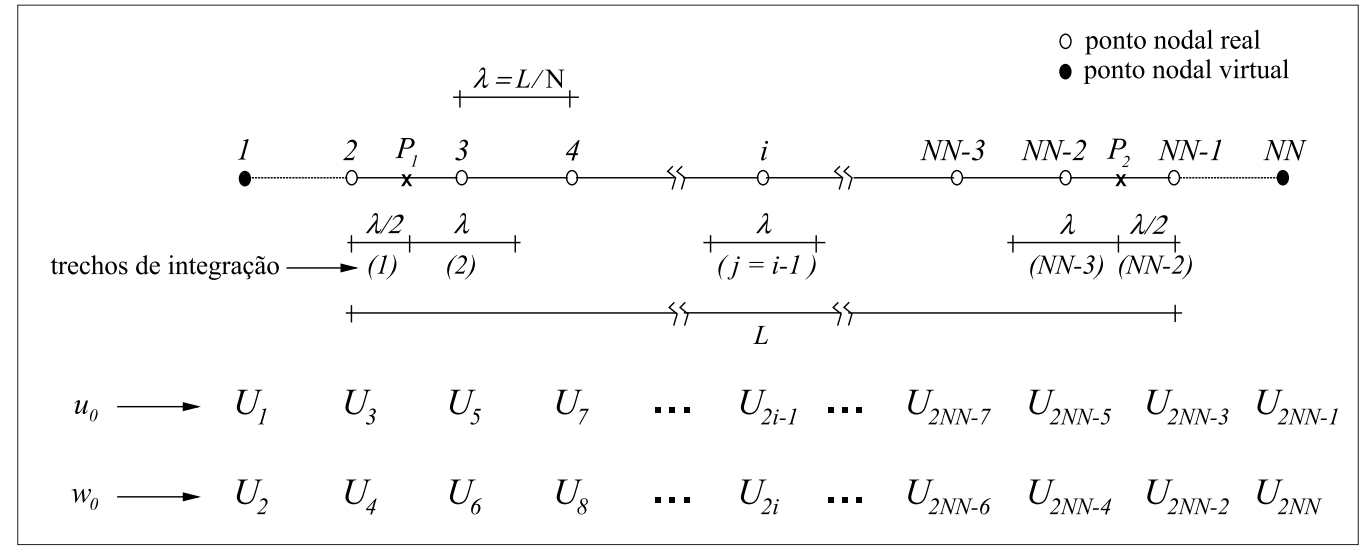

Figura 5 - Discretização da barra e sistema de numeração global para os deslocamentos nodais 
Na Figura 6 acha-se explicitado o sistema de numeração local para os deslocamentos nodais, com o objetivo de facilitar o cômputo da contribuição de cada trecho de integração no cálculo do trabalho virtual interno.

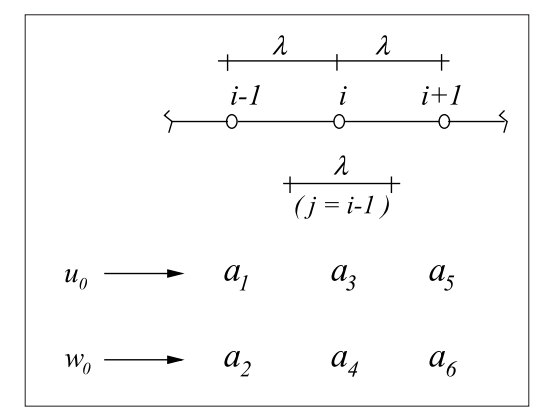

Figura 6 - Sistema de numeração local para os deslocamentos nodais

Nesse sistema define-se, em associação a três nós consecutivos $i-1 \quad i \quad i+1$, um conjunto de seis deslocamentos, $a_{1}$ a $a_{6}$, relacionados a $u_{0}$ e $w_{0}$, conforme indicado na Figura 6. Esses deslocamentos são ligados aos deslocamentos no sistema de numeração global mediante a seguinte relação, inerente a um trecho de integração $j$ :

$$
a_{k} \rightarrow U_{2 j+k-2}(k=1 \text { a } 6)
$$

Para avaliação das derivadas primeira dos deslocamentos em diferenças finitas serão consideradas a representação convencional (centrada) e reduzida. A representação centrada, avaliada no ponto pivotal $i$, é expressa por:

$$
f_{i}^{\prime}=\frac{1}{2 \lambda}\left(f_{i+1}-f_{i-1}\right)
$$

onde $f(x)$ representa os deslocamentos $w_{0}(x)$, sendo empregada nos $N N-2$ trechos de integração. Essa representação será também utilizada para a condição de contorno $\frac{\partial w_{0}}{\partial x}$, nas extremidades da barra. Quanto à derivada primeira de $u_{0}(x)$ nos trechos extremos de integração $(1)$ e $(N N-2)$ usar-se-á a representação reduzida:

$$
\begin{gathered}
f_{P_{1}}^{\prime}=\frac{1}{\lambda}\left(f_{3}-f_{2}\right) \\
f_{P_{2}}^{\prime}=\frac{1}{\lambda}\left(f_{N N-1}-f_{N N-2}\right)
\end{gathered}
$$


observando-se que $P_{1}$ e $P_{2}$ não são os pontos médios dos correspondentes trechos de integração (ver Figura 5). Nos demais trechos a derivada primeira de $u_{0}(x)$ será avaliada na forma convencional (equação 20). Essa estratégia diferenciada para calcular $\frac{\partial u_{0}}{\partial x}$ evita a ocorrência de singularidade no sistema de equações algébricas, quando se utiliza apenas a representação convencional ao longo do domínio da barra (BORGES, 1994; LIMA, 1995).

Para a derivada segunda dos deslocamentos, adota-se apenas a forma convencional, dada por:

$$
f_{i}^{\prime \prime}=\frac{1}{\lambda^{2}}\left(f_{i+1}-2 f_{i}+f_{i-1}\right)
$$

\subsection{Avaliação das parcelas do Trabalho Virtual}

A avaliação numérica do trabalho virtual interno $\delta W_{\text {int }}$ é expressa pelo somatório:

onde

$$
\delta W_{i n t}=\sum_{j=1}^{N N-2} \delta W_{i n t}(j)
$$

$$
\delta W_{\text {int }}(j)=\sum_{p=1}^{6} \sum_{q=1}^{6} C(p, q) U_{2 j+q-2} \delta U_{2 j+q-2}
$$

Nessa equação, cabe observar que de acordo (14) os termos $C(p q)$ são funções das propriedades mecânicas e geométricas da barra, bem como do dano, sendo ainda funções do espaçamento nodal $\lambda$, por conta da utilização das representações (20), (21) e (22) para as derivadas primeira e segunda dos deslocamentos.

Para facilitar, o cômputo de $\delta W_{i n t}$ os termos $C(p q)$ foram reunidos como coeficientes das seguintes matrizes: 


$$
[C(p, q)]=\left[\begin{array}{cccccc}
0 & 0 & 0 & 0 & 0 & 0 \\
0 & \frac{D_{11}}{2 \lambda^{3}} & \frac{B_{11}}{2 \lambda^{2}} & -\frac{D_{11}}{\lambda^{3}} & -\frac{B_{11}}{2 \lambda^{2}} & \frac{D_{11}}{2 \lambda^{3}} \\
0 & \frac{B_{11}}{2 \lambda^{2}} & \frac{A_{11}}{2 \lambda} & -\frac{B_{11}}{\lambda^{2}} & -\frac{A_{11}}{2 \lambda} & \frac{B_{11}}{2 \lambda^{2}} \\
0 & -\frac{D_{11}}{\lambda^{3}} & -\frac{B_{11}}{\lambda^{2}} & \frac{2 D_{11}}{\lambda^{3}} & \frac{B_{11}}{\lambda^{2}} & -\frac{D_{11}}{\lambda^{3}} \\
0 & -\frac{B_{11}}{2 \lambda^{2}} & -\frac{A_{11}}{2 \lambda} & \frac{B_{11}}{\lambda^{2}} & \frac{A_{11}}{2 \lambda} & -\frac{B_{11}}{2 \lambda^{2}} \\
0 & \frac{D_{11}}{2 \lambda^{3}} & \frac{B_{11}}{2 \lambda^{2}} & -\frac{D_{11}}{\lambda^{3}} & -\frac{B_{11}}{2 \lambda^{2}} & \frac{D_{11}}{2 \lambda^{3}}
\end{array}\right], \text { para } j=1
$$

(25.b) $[C(p, q)]=\left[\begin{array}{cccccc}\frac{A_{11}}{4 \lambda} & \frac{B_{11}}{2 \lambda^{2}} & 0 & -\frac{B_{11}}{\lambda^{2}} & -\frac{A_{11}}{4 \lambda} & \frac{B_{11}}{2 \lambda^{2}} \\ \frac{B_{11}}{2 \lambda^{2}} & \frac{D_{11}}{\lambda^{3}} & 0 & -\frac{2 D_{11}}{\lambda^{3}} & -\frac{B_{11}}{2 \lambda^{2}} & \frac{D_{11}}{\lambda^{3}} \\ 0 & 0 & 0 & 0 & 0 & 0 \\ -\frac{B_{11}}{\lambda^{2}} & -\frac{2 D_{11}}{\lambda^{3}} & 0 & \frac{4 D_{11}}{\lambda^{3}} & \frac{B_{11}}{\lambda^{2}} & -\frac{2 D_{11}}{\lambda^{3}} \\ -\frac{A_{11}}{4 \lambda} & -\frac{B_{11}}{2 \lambda^{2}} & 0 & \frac{B_{11}}{\lambda^{2}} & \frac{A_{11}}{4 \lambda} & -\frac{B_{11}}{2 \lambda^{2}} \\ \frac{B_{11}}{2 \lambda^{2}} & \frac{D_{11}}{\lambda^{3}} & 0 & -\frac{2 D_{11}}{\lambda^{3}} & -\frac{B_{11}}{2 \lambda^{2}} & \frac{D_{11}}{\lambda^{3}}\end{array}\right]$ para $1<j<N \quad-2$

$$
[C(p, q)]=\left[\begin{array}{cccccc}
\frac{A_{11}}{2 \lambda} & \frac{B_{11}}{2 \lambda^{2}} & -\frac{A_{11}}{2 \lambda} & -\frac{B_{11}}{\lambda^{2}} & 0 & \frac{B_{11}}{2 \lambda^{2}} \\
\frac{B_{11}}{2 \lambda^{2}} & \frac{D_{11}}{2 \lambda^{3}} & -\frac{B_{11}}{2 \lambda^{2}} & -\frac{D_{11}}{\lambda^{3}} & 0 & \frac{D_{11}}{2 \lambda^{3}} \\
-\frac{A_{11}}{2 \lambda} & -\frac{B_{11}}{2 \lambda^{2}} & \frac{A_{11}}{2 \lambda} & -\frac{B_{11}}{\lambda^{2}} & 0 & -\frac{B_{11}}{2 \lambda^{2}} \\
-\frac{B_{11}}{\lambda^{2}} & -\frac{D_{11}}{\lambda^{3}} & \frac{B_{11}}{\lambda^{2}} & \frac{2 D_{11}}{\lambda^{3}} & 0 & -\frac{D_{11}}{\lambda^{3}} \\
0 & 0 & 0 & 0 & 0 & 0 \\
\frac{B_{11}}{2 \lambda^{2}} & \frac{D_{11}}{2 \lambda^{3}} & -\frac{B_{11}}{2 \lambda^{2}} & -\frac{D_{11}}{\lambda^{3}} & 0 & \frac{D_{11}}{2 \lambda^{3}}
\end{array}\right], \text { para } j=N \quad-2
$$

A avaliação numérica do trabalho virtual externo, tal como no caso de $\delta W_{i n t}$, é feita a partir do somatório das contribuições dos diversos trechos de integração considerados na barra: 


$$
\begin{aligned}
& \delta W_{e x t}=\sum_{j=1}^{N N-2}\left[p(j) \delta U_{2 j+1}+q(j) \delta U_{2 j+2}\right] \lambda_{j}+ \\
& +\bar{F}_{x L} \delta U_{2 N N-3}-\bar{F}_{x 0} \delta U_{3}+\bar{F}_{z L} \delta U_{2 N N-2}-\bar{F}_{z 0} \delta U_{4}- \\
& -\frac{\bar{M}_{L}}{2 \lambda} \delta U_{2 N N}+\frac{\bar{M}_{L}}{2 \lambda} \delta U_{2 N N-4}+\frac{\bar{M}_{0}}{2 \lambda} \delta U_{6}-\frac{\bar{M}_{0}}{2 \lambda} \delta U_{2}
\end{aligned}
$$

onde $p(j)$ e $q(j)$ são as cargas de domínio, supostas constantes ao longo do trecho de integração, e $F_{t}, \bar{F}_{x 0}, \bar{F}_{t}, \bar{F}_{z 0}, \bar{M}_{L}$ e $\bar{M}_{0}$ são as cargas de extremidade.

\subsection{Análise não linear}

Da igualdade entre as equações (24) e (26) (PTV) e a posterior imposição das condições de contorno cinemáticas do problema em estudo, surge um sistema de $2 N N$ equações lineares algébricas do tipo:

$$
[K]\{u\}=\{R\}
$$

onde $[K]$ é uma matriz quadrada, formada a partir das contribuições das matrizes (25), $\{R\}$ é o vetor de cargas, oriundo de (26), e $\{u\}$ é o vetor de deslocamentos contendo os $2 N N$ deslocamentos nodais (Figura 5). Observando que a partir da danificação do concreto é introduzida a não linearidade física em (27), através da atualização da matriz $[K]$, foi estabelecida uma estratégia de solução incremental-iterativa de Newton-Raphson e também de Controle de Deslocamentos, visando abranger problemas com a presença de pontos limites de carga, na relação carga-deslocamento.

\section{Estudo de caso}

\subsection{Resultado Experimental}

Para validação do modelo proposto foram analisados os resultados experimentais de Nunes e Agopyan (1998), que determinaram em laboratório o comportamento em flexão de vigas de concreto reforçadas com fibras de aço $(\mathrm{Lf}=49 \mathrm{~mm}$ e fator de forma $=$ $48,5)$, com diferentes teores de adição: $20 \mathrm{~kg} / \mathrm{m}^{3}, 40 \mathrm{~kg} / \mathrm{m}^{3}$ e $60 \mathrm{~kg} / \mathrm{m}^{3}$.

Para cada mistura, foram moldados quatro corpos-de-prova prismáticos com dimensões de ( $150 \times 150$ x 500) mm que foram ensaiados aos 28 dias na flexão com uma taxa de deslocamento de 0,5 mm/min, de acordo com a norma ASTM C1018 (ASTM, 
1992). O deslocamento vertical foi medido através de dois transdutores de deslocamento de variação linear (LVDT) posicionados nos dois lados do prisma através de um suporte metálico chamado "Yoke" (Figura 7a). As curvas carga-deslocamento obtidas são apresentadas na Figura 7b. Corpos-de-prova cilíndricos com $150 \mathrm{~mm}$ de diâmetro por $300 \mathrm{~mm}$ de altura foram utilizados para ensaio de resistência a compressão. Os resultados encontram-se na Tabela 1.

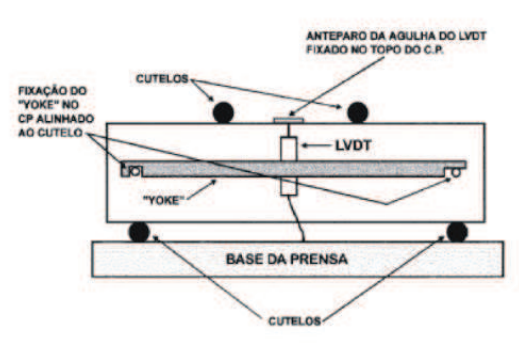

a)

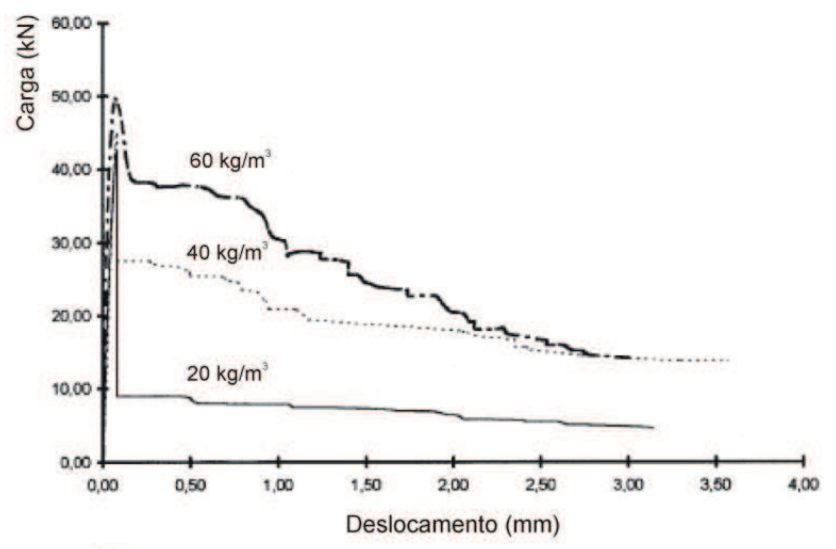

b)

Figura 7 - Resultados experimentais (Nunes e Agopyan, 1998): a) configuração do ensaio; b) curva carga-deslocamento.

Tabela 1 - Propriedade dos concretos

\begin{tabular}{cccccc}
\hline \multirow{2}{*}{ Teor de fibras $\left(\mathrm{kg} / \mathrm{m}^{3}\right)$} & \multicolumn{2}{c}{ Valores Experimentais } & \multicolumn{3}{c}{ Valores teóricos } \\
& $\mathrm{fc}(\mathrm{MPa})$ & $\mathrm{ft}(\mathrm{MPa})$ & $\mathrm{E}(\mathrm{GPa})$ & $v$ & $\varepsilon(\mathrm{mm} / \mathrm{mm})$ \\
\hline $20 \mathrm{~kg} / \mathrm{m}^{3}$ & 39,19 & 5,81 & 32,27 & & \\
$40 \mathrm{~kg} / \mathrm{m}^{3}$ & 40,64 & 6,81 & 32,66 & 0,2 & 0,0022 \\
$60 \mathrm{~kg} / \mathrm{m}^{3}$ & 40,40 & 6,76 & 32,60 & & \\
\hline
\end{tabular}

Para avaliação numérica são necessários o módulo de elasticidade (E), a deformação de pico e o coeficiente de Poisson. Como não foram determinadas experimentalmente curvas tensão-deformação na compressão, o valor de $\mathrm{E}$ foi determinado pela equação $\mathrm{E}=9500 \mathrm{f}_{\mathrm{c}}^{\mathrm{T} 33}$,proposta pelo Eurocode 2 (1991), o coeficiente de Poisson foi adotado como 0,2 e o valor da deformação de pico foi adotado igual a $0,022 \mathrm{~mm} / \mathrm{mm}$, de acordo com 0 MC90 (CEB-FIP, 1991). Esses resultados estão também na Tabela 1. 


\subsection{Curvas tensão-deformação teóricas}

A partir das propriedades apresentadas na Tabela 1 foram determinados os diagramas tensão-deformação teóricos. Na compressão utilizou-se o modelo não linear baseado na Mecânica do Dano (Mazars e Lamaitre, 1984) e apresentado no item 2.1, com valores de $\mathrm{A}_{\mathrm{c}}$ e $\mathrm{B}_{\mathrm{c}}$, apresentados na Tabela 2, que resultassem em curvas com as mesmas propriedades apresentadas na Tabela 1.

Na tração foram utilizados três modelos: i) modelo não-linear baseado na Mecânica do Dano (Mazars e Lamaitre, 1984), denominado como Modelo Proposto; ii) Modelo Linearizado com tensão pós-fissuração constante, mostrado na Figura $1 \mathrm{c}$, com $\sigma_{2}=$ $\sigma_{3}$; iii) Modelo Trilinear com tensão pós-fissuração descendente, mostrado na Figura $1 \mathrm{c}, \operatorname{com} \sigma_{3}=0$.

A utilização dos modelos Não-linear e Linearizado foi realizada com o objetivo de avaliar a influência da não-linearidade do diagrama tensão-deformação na tração sobre o comportamento na flexão. O modelo Trlinear foi utilizado por Grossi (2006) para modelagem dos mesmos resultados experimentais, mas utilizando o Método dos Elementos Finitos em uma formulação bidimensional, e servirá como referência para comparação com o Método das Diferenças Finitas Energéticas, aqui empregado. Como não foram realizados ensaios de tração direta que permitissem a determinação experimental dos parâmetros das curvas, estes foram obtidos a partir dos resultados do ensaio de flexão, por análise inversa. Portanto, na Tabela 2 foram incluídos os parâmetros de tração determinados para o modelo não linear, e na Tabela 3 são apresentados os parâmetros teóricos utilizados nas modelagens linearizadas do diagrama tensão-deformação na tração.

Tabela 2 - Parâmetros para modelagem dos diagramas tensão-deformação não-lineares

\begin{tabular}{c|c|c|c|c|c}
\hline \multirow{2}{*}{ Teor de fibras $\left(\mathrm{kg} / \mathrm{m}^{3}\right)$} & \multirow{2}{*}{$\begin{array}{c}\varepsilon_{\mathrm{do}}=\mathrm{f}_{\mathrm{f}} / \mathrm{E} \\
\left(10^{-4}\right)\end{array}$} & \multicolumn{2}{|c|}{ Compressão } & \multicolumn{2}{|c}{ Tração } \\
\cline { 3 - 6 } & & Ac & Bc & At & $\mathrm{Bt}$ \\
\hline $20 \mathrm{~kg} / \mathrm{m}^{3}$ & 1,801 & 1,33 & 1643 & 0,95 & $5 \times 104$ \\
$40 \mathrm{~kg} / \mathrm{m}^{3}$ & 2,085 & 1,33 & 1643 & 0,84 & $5 \times 104$ \\
$60 \mathrm{~kg} / \mathrm{m}^{3}$ & 2,074 & 1,33 & 1643 & 0,73 & $5 \times 104$ \\
\hline
\end{tabular}

Tabela 3 - Parâmetros para modelagem dos diagramas tensão-deformação lineares, na tração

\begin{tabular}{c|c|c|c|c|c|c|c}
\hline & & & & & MLC & MLD \\
\cline { 5 - 8 } $\begin{array}{c}\text { Teor de fibras } \\
\left(\mathrm{kg} / \mathrm{m}^{3}\right)\end{array}$ & $\varepsilon_{1}=\mathrm{f}_{\mathrm{t}} / \mathrm{E}$ & $\begin{array}{c}\sigma_{1}=\mathrm{f}_{\mathrm{t}} \\
\text { (MPa) }\end{array}$ & $\varepsilon_{2}$ & $\begin{array}{c}\sigma_{2} \\
(\mathrm{MPa})\end{array}$ & $\varepsilon_{3}$ & $\begin{array}{c}\sigma_{3}=\sigma_{2} \\
(\mathrm{MPa})\end{array}$ & $\begin{array}{c}\sigma_{3} \\
\text { (MPa) }\end{array}$ \\
\hline $20 \mathrm{~kg} / \mathrm{m}^{3}$ & $1,801 \times 10-4$ & 5,81 & $1,891 \times 10-4$ & 0,29 & 0,841 & 0,29 & 0 \\
$40 \mathrm{~kg} / \mathrm{m}^{3}$ & $2,085 \times 10-4$ & 6,81 & $2,189 \times 10-4$ & 1,09 & 0,191 & 1,09 & 0 \\
$60 \mathrm{~kg} / \mathrm{m}^{3}$ & $2,074 \times 10-4$ & 6,76 & $2,177 \times 10-4$ & 1,82 & 0,101 & 1,82 & 0 \\
\hline
\end{tabular}




\subsection{Modelagem Numérica}

Nas análises que se seguem, a partir do MDFE, para todas as vigas foi adotada uma discretização do comprimento em 12 subdivisões e da seção transversal em 20 camadas. Além disso, instituiu-se o passo de carga de $1,0 \mathrm{kN}$ e a tolerância $10^{-6}$ para 0 processo iterativo (controle de deslocamentos).

Nas Figuras 8 a 10 são apresentadas as curvas carga-deslocamento experimentais e as curvas numéricas obtidas com o MDFE, utilizando tensão-deformação não-linear (modelo proposto), e as curvas obtidas por Grossi (2006) pelo MEF, com o modelo trilinear.

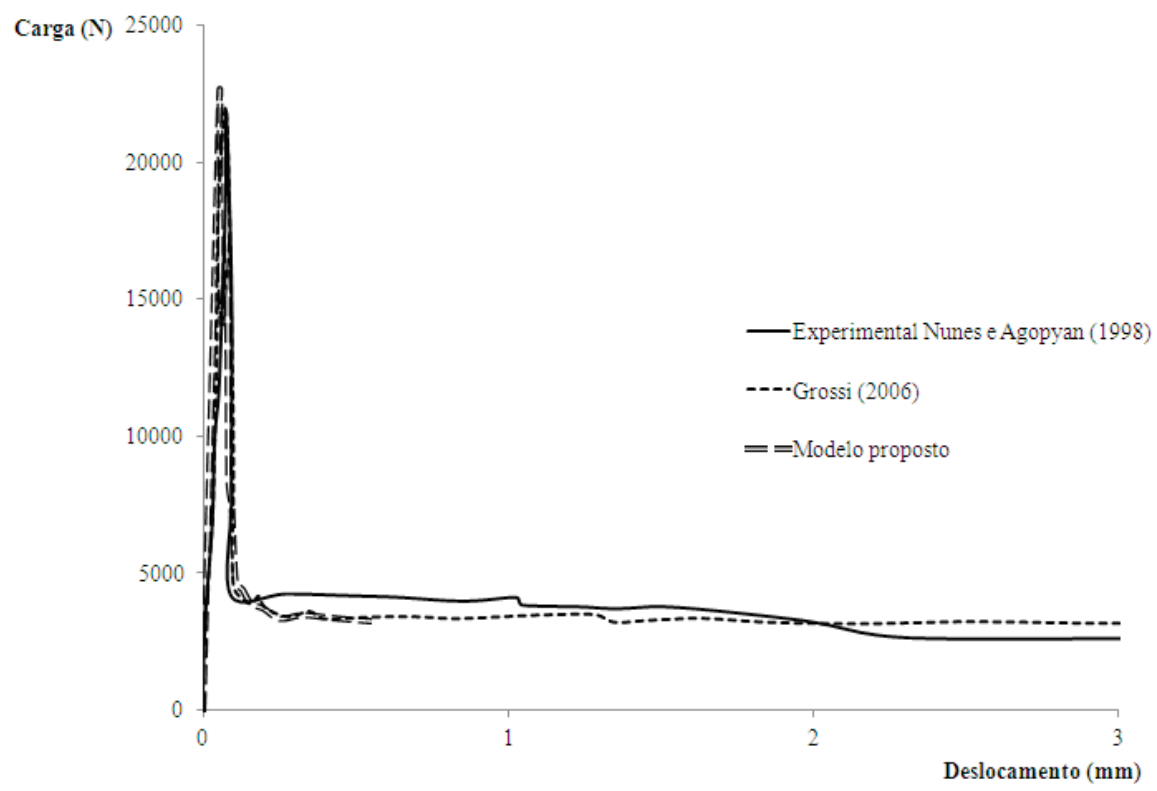

Figura 8 - Curvas carga-deslocamento experimental e teóricas para a viga de concreto com $20 \mathrm{~kg} / \mathrm{m}^{3}$ de fibras de aço 


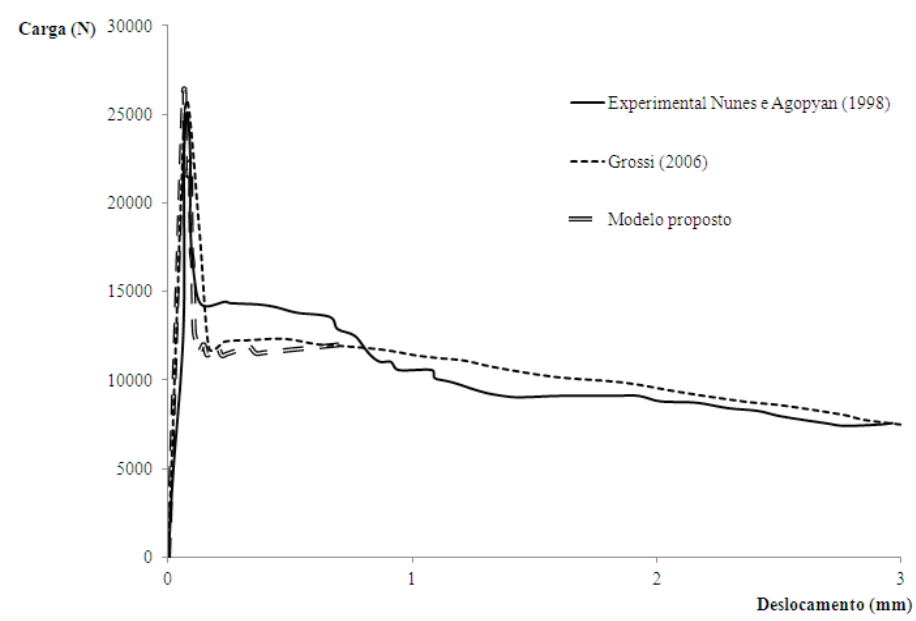

Figura 9 - Curvas carga-deslocamento experimental e teóricas para a viga de concreto com $40 \mathrm{~kg} / \mathrm{m}^{3}$ de fibras de aço

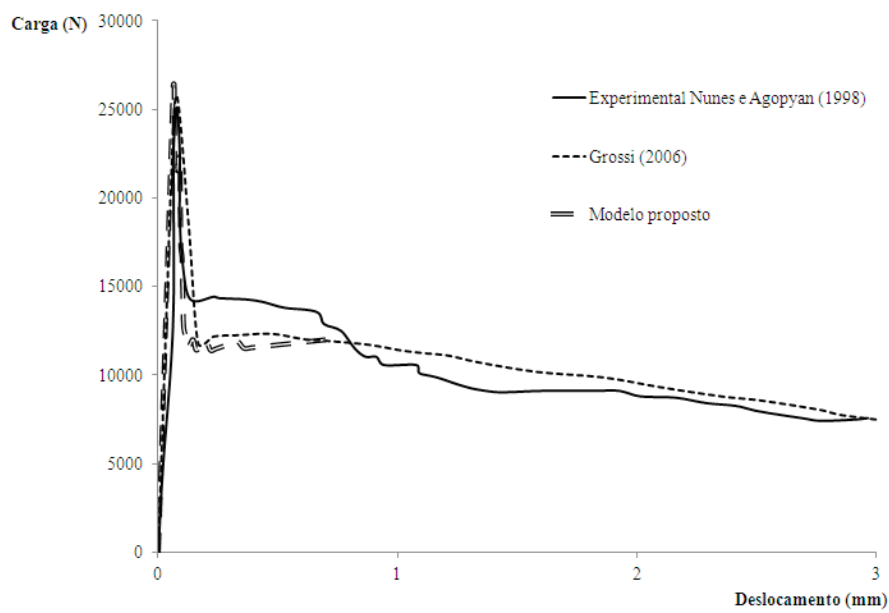

Figura 10 - Curvas carga-deslocamento experimental e teóricas para a viga de concreto com $60 \mathrm{~kg} / \mathrm{m}^{3}$ de fibras de aço

Analisando-se os resultados obtidos, constata-se boa concordância entre as curvas obtidas através do modelo proposto e as obtidas numericamente por Grossi (2006). Além disso, verifica-se uma boa representação das curvas experimentais. Entretanto, o modelo sugerido apresentou limitações: a maior vantagem da utilização de fibras de aço em concreto é o aumento da tenacidade no pós-pico, o que não foi possível alcançar com fidelidade, uma vez que a simulação numérica realizada finalizou a deslocamentos menores do que os registrados experimentalmente por Nunes e Agopyan (1998) e também por Grossi (2006), a partir de seu modelo bidimensional. 
Acredita-se que, devido a não-linearidade do modelo de dano, houve dificuldade de convergência numérica. Por isso, foi implementado o modelo linearizado com patamar horizontal de tensão, após o pico, com aproximadamente o mesmo valor do modelo proposto. Os resultados estão apresentados nas Figuras 11 a 13.

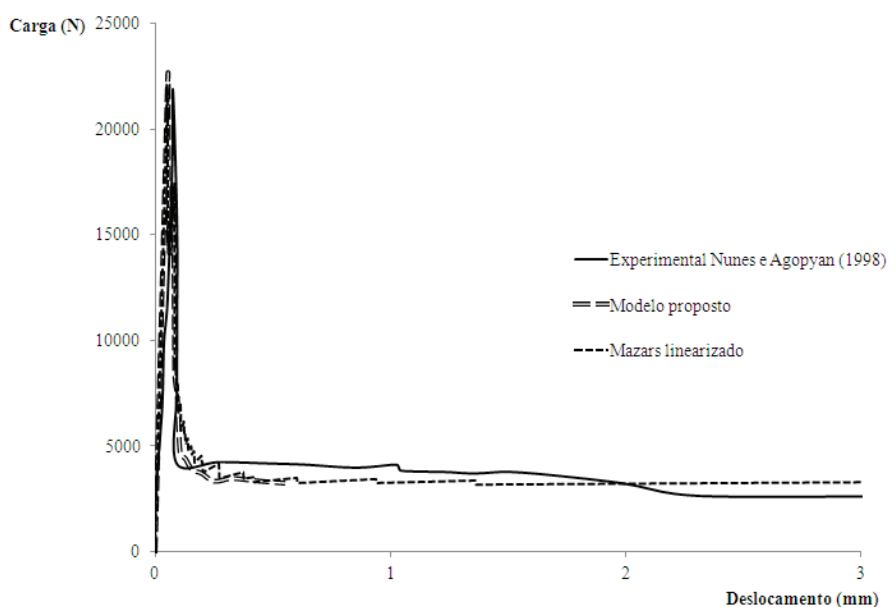

Figura 11 - Curvas carga-deslocamento experimental e teóricas ((Mazars e Lamaitre, 1984)) para a viga de concreto com $20 \mathrm{~kg} / \mathrm{m}^{3}$ de fibras de aço

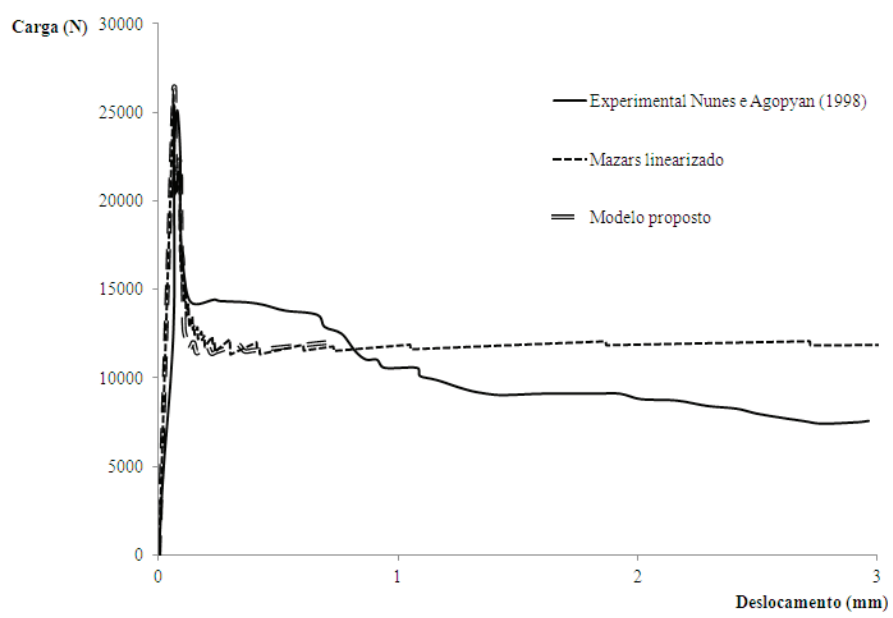

Figura 12 - Curvas carga-deslocamento experimental e teóricas ((Mazars e Lamaitre, 1984)) para a viga de concreto com $40 \mathrm{~kg} / \mathrm{m}^{3}$ de fibras de aço 


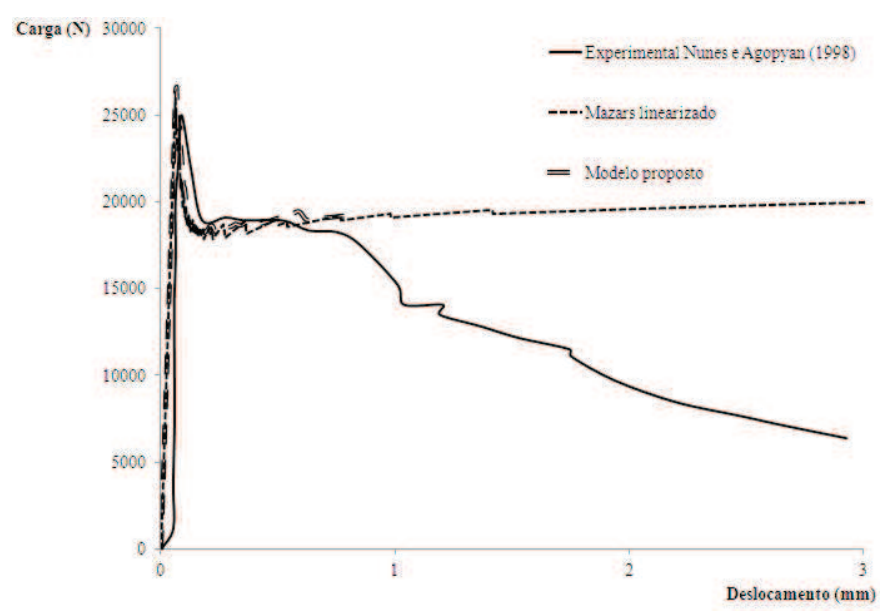

Figura 13 - Curvas carga-deslocamento experimental e teóricas ((Mazars e Lamaitre, 1984)) para a viga de concreto com $60 \mathrm{~kg} / \mathrm{m}^{3}$ de fibras de aço

Nessas curvas verifica-se que há uma convergência quando se adota o modelo linearizado para o diagrama de tração de Mazars e Lamaitre (1984). No entanto, comparando-se a curva obtida com a experimental é identificado um maior valor de carga pós-fissuração para as vigas de $40 \mathrm{~kg} / \mathrm{m}^{3}$ e $60 \mathrm{~kg} / \mathrm{m}^{3}$ à medida que o deslocamento aumenta, provavelmente em razão da maior tensão pós-pico observada no diagrama de tração linearizado adotado. De fato, tanto a curva carga-deslocamento como a curva tensão-deformação do material apresentam certa plastificação após a primeira fissura. Por isso, foi implementado, no MDFE, o diagrama trilinear utilizado por Grossi (2006), como mostram as Figuras 14 a 16, cuja tensão pós-pico tende a zero na deformação máxima $\left(\varepsilon_{3}\right)$. 


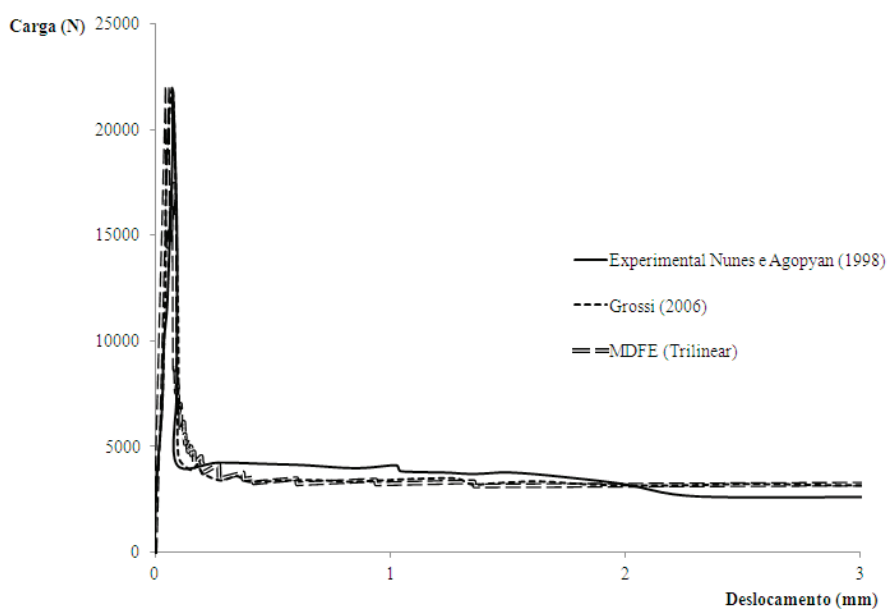

Figura 14 - Resultado do MDFE (trilinear) aplicado ao concreto com $20 \mathrm{~kg} / \mathrm{m}^{3}$ de fibras de aço

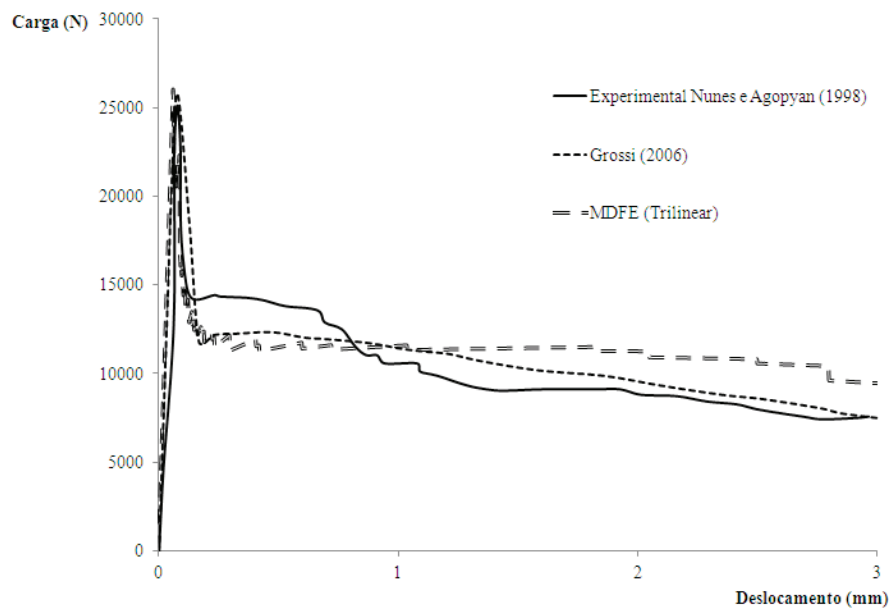

Figura 15 - Resultado do MDFE (trilinear) aplicado ao concreto com $40 \mathrm{~kg} / \mathrm{m}^{3}$ de fibras de aço 


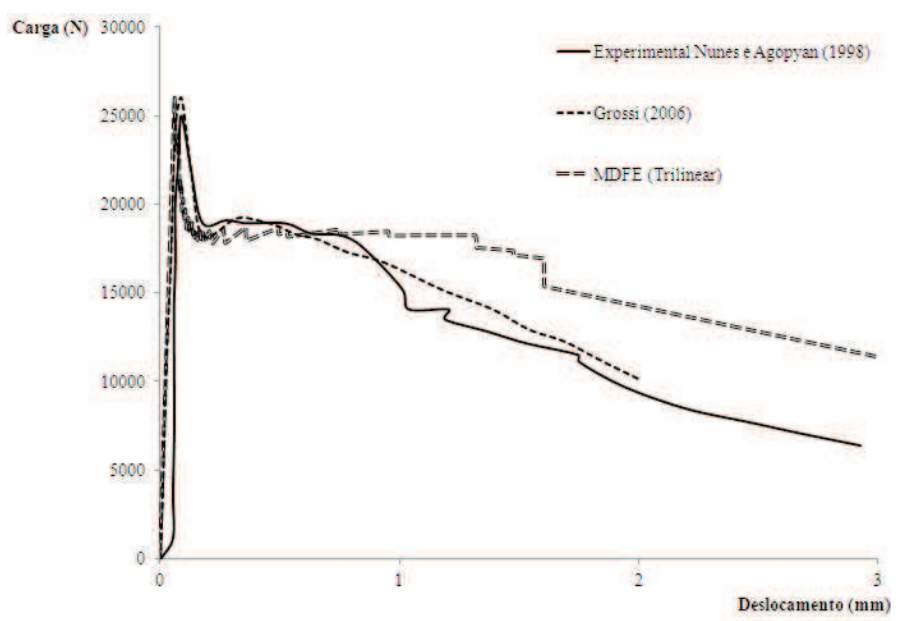

Figura 16 - Resultado do MDFE (trilinear) aplicado ao concreto com $60 \mathrm{~kg} / \mathrm{m}^{3}$ de fibras de aço

Verifica-se que há aproximação entre o comportamento das curvas obtidas com o modelo trilinear inserido no programa com o MDFE e o comportamento das curvas de Grossi (2006), que utilizou o MEF. Além disso, nota-se que os deslocamentos atingem valores da ordem do experimental. Entretanto, foi observado que, para os concretos, cujos teores de fibras de aço utilizadas são $40 \mathrm{~kg} / \mathrm{m}^{3}$ e $60 \mathrm{~kg} / \mathrm{m}^{3}$, existiu uma diferença entre os resultados numéricos de Grossi $\left(20{ }^{\circ}\right)$ e os calculados neste trabalho. Acredita-se que essa diferença é consequência da ${ }^{\varepsilon_{3}}$ utilizada, visto que Grossi (2006) não apresentou os valores utilizados.

\section{Conclusão}

No presente trabalho foi proposta uma formulação teórico-computacional para prever o comportamento à flexão de vigas constituídas por CRFA, com armadura convencional ou não, que se valeu do modelo de dano de (Mazars e Lamaitre, 1984) para descrever a perda de rigidez do concreto, e cujo tratamento numérico utilizou a teoria clássica dos laminados e o MDFE.

Para validar esse modelo de análise, inicialmente foram realizados estudos paramétricos visando a simulação do comportamento a flexão de vigas não armadas reforçadas com três teores de fibra de aço. Foram testados três tipos de digramas tensão-deformação na tração: um não-linear e dois lineares.

\section{Agradecimentos}

A FAPESB/CNPQ pelo apoio financeiro (Projeto PPP0010/2010) e a FAPESB pela Bolsa de Mestrado oferecida ao primeiro autor. 


\section{Referências}

American Society for Testing and Materials, ASTM C 1018 (1992), Standanrt Test Method foe Flexural Toughness and First-Crack Strength of Fibre-Reinforced Concrete (Using beam with Third Point-Loading), Annual Book of ASTM Standards, Vol. 04.02, ASTM, Philadelphia.

Associação Brasileira de Normas Técnicas - ABNT (1995). NBR 6118: Projeto de estruturas de aço e de estruturas mistas de aço e concreto de edifícios. Rio de Janeiro.

Barros, J. A. (1995). O. Comportamento do betão reforçado com fibras: Análise experimental e simulação numérica. Porto, 1995. Tese de Doutoramento, Universidade do Porto, Portugal.

Bentur, A.; Mindess, S. (2007). Fibre reinforced cementitious composites. 2. Ed. Modern Concrete Technology Series. Taylor \& Francis.

Borges, M.S.S. (1994). Flexo-torção em hastes de paredes delgadas de seção aberta com abordagem por diferenças finitas. Rio de Janeiro Dissertação de Mestrado, Universidade Federal do Rio de Janeiro.

Committee Euro-international du Beton (CEB) \& Federation International de la Précontrainte (FIP). (1991). Model Code 1990 - Design code, Lausanne: Thomas Telford Services Ltd.

Daniel, I.M., and Ishai, O. (2006). Engineering mechanics of composite materials. Oxford University Press, Inc..

EUROCODE 2 (ENV 1992-1-1) (1991). Eurocode 2: Design of concrete structures - Part 1: general rules and rules of construction.

Furlan Jr.; Hana, J. (1997). Shear Behaviour of Fiber Reinforced Beams Concrete. Cement and Concrete Composites, 19, p. 359-366.

Grossi, B. F. (2006). Uma contribuição para a modelagem numérica do concreto com fibras curtas de aço. Belo Horizonte, 2006. Tese de Doutorado, Universidade Federal de Minas Gerais.

Habibi, A.R., Moharrami, H. (2010). Concrete frames, Finite Elements in Analysis and Design, 46, p.571-584.

Jones, R.M. (1999). Mechanics of composite materials. Taylor \& Francis Group.

Jones, J.; Wua, C.; Oehlers, D. J.; Whittaker, A. S.; Sun, W.; Marks, S.; Coppola, R. (2009). Finite difference analysis of simply supported RC slabs for blast loadings. Engineering Structures, 31, p. 2825-2832.

Lima, J. M. F. (1995). Análise dinâmica da flexão de barras com não-linearidade geométrica utilizando diferenças finitas energéticas. Rio de Janeiro, 1995. Dissertação de Mestrado, Universidade Federal do Rio de Janeiro.

Massicote, B. (2003). Structural use of SFRC: examples of possible applications. In: Dimensionamento de Estruturas de Betão Reforçados com fibras de aço. Guimarães: UMinho.

Mazars, J., and Lemaitre, J. (1984). Application of Continuous Damage Mechanics to Strain and Fracture Behavior of Concrete, In: SHAH, S.P. editor. USA: Northwestern University.

Naaman, A.E., Reinhardt, H.W. (2006) Proposed classification of HPFRC composites based on their tensile response. Materials and structures, 39, p.547-555.

Nataraja, M. C.; Dhang, N.; Gupta, A. P. (1999). Stress-strain curves for steel-fiber reinforced concrete under compression. Cement and Concrete Composites, 21, p. 383-390. 
Nunes, N. L.; Agopyan, V. (1998). A influência do fator de forma da fibra na tenacidade à flexão do concreto reforçado com fibras de aço. 18 p. Boletim Técnico BT/PCC/225, Departamento de Engenharia de Construção Civil, Escola Politécnica da Universidade de São Paulo (USP), São Paulo, SP.

Reddy, J.N. (2004). Mechanics of laminated composite plates and shells: theory and analysis. CRC Press.

RILEM TC 162-TDF (2002). Test and design methods for steel fibre reinforced concrete. Matériaux et Constructions, 35, p.262-278.

Ruano, G.. Isla, F.; Luccioni, B. (2012). Resistencia al corte de vigas de hormigón armado reparadas con hormigón reforzado con fibras de acero. Revista Sul-Americana de Engenharia Estrutural. Passo Fundo, 9 (2), p.41-64.

Soranakom, C.; Mobasher, B. (2007). Closed-form solutions for flexural response of fiber-reinforced concrete beams. Journal of Engeneering Mechanics, p. 933-941. 


\title{
Energy Finite Difference Method for simulation of flexural behavior of steel fibers reinforced concrete
}

\begin{abstract}
The use of steel fibers as reinforcement of structural concrete had increased in recent years, however the development of theoretical or computational models is still incipient. The addition of steel fibers modifying stress-strain behavior of the concrete in both compression and tension, and, in the latter case, the material retains a residual stress after cracking, even at high deformations. Therefore, it becomes necessary to use different stress-strain diagrams incorporating the contribution of steel fibers to more accurately predict the structural behavior of these elements. In this paper we present a variational formulation based on the Energy Finite Difference Method for predicting the flexural behavior of steel fiber reinforced concrete beams, combining the classical theory of laminates (TCL) with a damage model. The validation of the proposal is based on experimental and theoretical load-displacement curves found in the literature for reinforced concrete beams with three levels of reinforcing steel fibers.
\end{abstract}

\section{Conclusion}

It was verified a difficulty of the non-linear model, based on Damage Mechanics, to obtain the displacements achieved experimentally. This problem was solved by linearization of this tensile curve. To obtain a better agreement with experimental results, a modification of tensile curve it was applied with reduction of residual post-cracking stress. The comparison of Energy Finite Difference Model with results from a TwoDimensional Finite Element Model, developed by Grossi (2006), showed similarity in predicting the load-displacement behavior (post-peak) when the stress-strain diagram trilinear was used. 